Received: November 8, 1990

[1] a) H. Buff, F. Wohler, Amm. 1857, 104, 94; b) A Ladenburg. Ber. 1873, 6, 379.

[2] J. A. Meads, F. S. Kipping, J. Am. Chem. Soc 1914, $105,679$.

[3] D. W. Scott, J. Am. Chem. Soc 1946, 68, 356.

14] M. G. Voronkov, V. I. Lavrent'yev, Topics Curr Chem. 1982, 102, 199.

[5] D. Herren, H. Bürgy, G. Calzaferri, Helv. Chim Acta 1991, 74, 24.

[6] M. M. Levitsky, O. I. Schegolikhina, A. A Zhdanov, V. A. Igonin, Yu. E. Ovchinnikov, V.E Shklover, Yu. T. Struchkov,J.Organomet. Chem. (Russ.), in press.

[7] C. L. Frye, W. T. Collins, J.Am. Chem.Soc. 1970, 92, 5586 .

[8] H. Bürgy, G. Calzaferri,J.Chromatogr.1990,507, 481.

19] P. A. Agaskar, submitted to I. Am. Chem. Soc.

[10] F.J. Feher, K.J. Weller, Organometallics 1990, 9 , 2638.

[11] F.J. Feher, K.J. Weller, submitted to Inorg. Chem

[12] a) F. J. Feher, J. F. Walzer, Inorg. Chem., in press; b) F. J. Feher, J. F. Walzer, R. L. Blanski, submitted to J. Am. Chem. Soc.

[13] F. J. Feher, R. L. Blanski, J. Chem. Soc., Chem Commun., in press.

[14] K.Olsson, C. Grönwall, Arkiu Kemi 1961, 17,529.

[15] F. J. Feher, T. A. Budzichowski, J. Organomet Chem. 1989, 373, 153.

[16] F. J. Feher, T. A. Budzichowski, J. Organomet Chem. 1989, 379, 33.

[17] V.W. Day, W. G. Klemperer, V. V. Mainz, D. M. Millar.J. Am. Chem. Soc. 1985, 107. 8262.

[18] a) P. A. Agaskar, J. Am. Chem. Soc. 1989, 111 , 6858; b) P. A. Agaskar, Inorg. Chem. 1990, 29 1603 .

[19] P.A.Agaskar, V. W. Day, W.G. Klemperer,J.Am. Chem. Soc. 1987, 109, 5554.
[20] H. Bürgy, G.Calzaferri, Helv.Chim.Acta 1990,73, 698.

[21] G. Calzaferri, R. Hoffmann,J.Chem.Soc., Dalton Trans., in press.

[22] 1. Ojima, "The Chemistry of Organic Silicon Compounds', Eds. S. Patai and Z. Rappoport, Wiley, New York, 1989, Part II, pp. 1479-1526, and ref. cit, therein.

[23] H. Bürgy, Doctoral Thesis, University of Bern, $199 \mathrm{~L}$.

[24] P. Bomhauser, G. Calzaferri, Spectrochim. Acta, Part A 1990, 46, 1045.

[25] M. Bärtsch, P. Bomhauser, G. Calzaferri, to be submitted.

[26] B. A. Kolesov, T. N. Martynova, T.I. Chupakhina, Zh. Strukt. 1988, 29, 77.

[27] F. J. Feher, D. A. Newman, J. F. Walzer, J. Am Chem. Soc. 1989, 1/1, 1741.

[28] E. J. J. Groenen, A. G. T. G. Kortbeeck, M. Mackay, O. Sudmeijer, Zeolites 1986, 6,403.

[29] E. J. J. Groenen, C. A. Emeis, J. P. Van den Berg, P. C. de Jong-Versloot, Zeolites 1987, 474.

[30] a) G. Calzaferri, M. Gori, Helv. Chim. Acta 1985 , 68,$1617 ;$ b) G. Calzaferri, M. Gori, H.R. Grüniger, W. Spahni, 'Proceedings Photoelectrochemistry: Fundamental Processes and Measurement 'Techniques, The Electrochemical Soc.', Eds. W. L. Wallace, A. J. Nozik, S. K. Deb, and R. H. Wilson, 1982, Vol.82-3, p. 264; c) H.R. Grüniger, G. Calzaferri, Helv. Chim. Acta 1979, 62, 2547.

[31] a) R. Beer, H. Bürgy, G. Calzaferri, I. Kamber, $J$ Electron. Spec. Rel. Phen. 1987, 44, 121; b) H. Bürgy, G. Calzaferri, 1. Kamber, Mikrochim. Acta 1988, 1, 401.

[32] N. J. Harrick, 'Internal Reflection Spectroscopy', John Wiley \& Sons, New York, 1967; F. M. Mirabella, Jr., N.J. Harrick, Harrick Scientific Corpor., Ossining, NY, 1985.
[33] J. Baumann, R. Beer, G. Calzaferri, B. Waldeck, J. Phys. Chem. 1989, 93, 2292.

[34] L. M. Hananaschvili, K. A. Adrianov, 'Technology of the Organoelement Monomers and Polymers, Chimia Moscow', 1983, pp. 293-295 (Russ.).

[35] W. M. Meier, D. H. Olson, Atlas of Zeolite Structure Types', 2nd edn., Butterworths, London, 1988.

[36] T. J. Meyer, Acc. Chem. Res. 1989, 22, 163.

[37] G. Calzaferri, L. Forss, W. Spahni, Chenic in unserer Zeit 1987, 2/, 161.

[38] a) G. Calzaferri, S. Hug, Th. Hugentobler, B. Sulzberger, J. Photochem. 1984, 26, 109; b) G. Calzaferri, W. Spahni, ibid. 1986, 32, 151; c) Chimia 1986, 40, 435; d) ibid. 1987, 41, 200

[39] G. Calzaferri, Proceedings of the 3 rd intemational summerschool, 'Solar Energy 88', Ed.G. Faninger, Kaprun/Austria, IFF, Klagenfurt, 1989, p. 119.

[40] A. Harriman, M. A. West, 'Photogeneration of Hydrogen', Academic Press, London, 1982.

[41] E. Amouyal, B. Zidler, Israel J. Chem. 1982, 22, 117.

[42] J. Hawecker, J.-M. Lehn, R.Ziessel,J.Chem.Soc., Chem. Commun. 1983, 536.

[43] G. Calzaferri, K. Hädener, J. Li, J. Chem. Soc., Chem. Commum., in press.

[44] G. Calzaferri, ESA SP-240 1985, 93.

[45] G. Calzaferri, J. Li, K. Hädener, N. Gfeller, D. Herren, M. Brändle, Eight International Conference on Photochemical Conversion and Storage of Solar Energy (I.P. S. 8), Palermo, Italy, 1990, Book of Abstracts, p. 212.
Chimia 45 (199/) 8-10

() Schweiz. Chemiker-Verband; ISSN 0009-4293

\title{
Bestimmung von Selen in Lebensmitteln und in Mineralien mittels Neutronenaktivierungs- analysen
}

\section{Marcel Langenauer und Urs Krähenbühl*}

Abstract. The determination of Se became important, since it was recognized that this trace element is essential for the human and animal diet. Lately, it was found that most patients suffering from a cancer disease manifest very low values of Se in their blood plasma. Activation analysis is a well suited technique to measure the concentrations of $S e$ in a vast range of materials with very different concentrations. At the lower end of concentrations, a radiochemical separation of Se following the irradiation is mandatory to obtain reliable results in the determination.

\section{Einleitung}

Selen galt seit Jangem als äusserst toxisches Element. Erst 1957 wurde seine essentielle Bedeutung für den Menschen erkannt
[1]. Durch Abgabe von Selentabletten an die Bevölkerung der Keshan-Region in China konnte die Kashin-Becksche Krankheit, eine rheumatisch-athritische Erkrankung, fast vollständig eliminiert werden. Heute ist be- kannt, dass bei vielen weiteren Krankheiten wie Krebs, Leukämie oder AIDS der SeGehalt im Blut einen Mangel aufzeigt. Es ist daher wichtig, dass die Bevölkerung genügend mit Se versorgt wird. Dieses Spurenelement kommt in Fleisch, Fisch und Getreideprodukten angereichert vor [2][3], wogegen es in Gemüse, Früchten und Milchprodukten verarmt ist [3]. Der tägliche Mindestbedarf des Menschen beträgt I $\mu \mathrm{g} \mathrm{Se} / \mathrm{kg}$ Körpergewicht [2]. Im allgemeinen sind die Böden der Schweiz recht arm an Se [4]. Importiertes Getreide aus den USA oder Kanada weist einen 10 bis 100 mal höheren Gehalt an diesem essentiellen Element auf als Weizen, der in der Schweiz geerntet wurde. Eine ausreichende Versorgung der Schweiz mit Se ist Dank der Getreideimporte gewährleistet. Die Entwicklung einer

*Korrespondenz: Prof. U. Krähenbühl Laboratorium für Radiochemie Universität Bern

Frejestrasse 3, CH-3000 Bern 9 
Received: November 8, 1990

[1] a) H. Buff, F. Wohler, Amm. 1857, 104, 94; b) A Ladenburg. Ber. 1873, 6, 379.

[2] J. A. Meads, F. S. Kipping, J. Am. Chem. Soc 1914, $105,679$.

[3] D. W. Scott, J. Am. Chem. Soc 1946, 68, 356.

14] M. G. Voronkov, V. I. Lavrent'yev, Topics Curr Chem. 1982, 102, 199.

[5] D. Herren, H. Bürgy, G. Calzaferri, Helv. Chim Acta 1991, 74, 24.

[6] M. M. Levitsky, O. I. Schegolikhina, A. A Zhdanov, V. A. Igonin, Yu. E. Ovchinnikov, V.E Shklover, Yu. T. Struchkov,J.Organomet. Chem. (Russ.), in press.

[7] C. L. Frye, W. T. Collins, J.Am. Chem.Soc. 1970, 92, 5586 .

[8] H. Bürgy, G. Calzaferri,J.Chromatogr.1990,507, 481.

19] P. A. Agaskar, submitted to I. Am. Chem. Soc.

[10] F.J. Feher, K.J. Weller, Organometallics 1990, 9 , 2638.

[11] F.J. Feher, K.J. Weller, submitted to Inorg. Chem

[12] a) F. J. Feher, J. F. Walzer, Inorg. Chem., in press; b) F. J. Feher, J. F. Walzer, R. L. Blanski, submitted to J. Am. Chem. Soc.

[13] F. J. Feher, R. L. Blanski, J. Chem. Soc., Chem Commun., in press.

[14] K.Olsson, C. Grönwall, Arkiu Kemi 1961, 17,529.

[15] F. J. Feher, T. A. Budzichowski, J. Organomet Chem. 1989, 373, 153.

[16] F. J. Feher, T. A. Budzichowski, J. Organomet Chem. 1989, 379, 33.

[17] V.W. Day, W. G. Klemperer, V. V. Mainz, D. M. Millar.J. Am. Chem. Soc. 1985, 107. 8262.

[18] a) P. A. Agaskar, J. Am. Chem. Soc. 1989, 111 , 6858; b) P. A. Agaskar, Inorg. Chem. 1990, 29 1603 .

[19] P.A.Agaskar, V. W. Day, W.G. Klemperer,J.Am. Chem. Soc. 1987, 109, 5554.
[20] H. Bürgy, G.Calzaferri, Helv.Chim.Acta 1990,73, 698.

[21] G. Calzaferri, R. Hoffmann,J.Chem.Soc., Dalton Trans., in press.

[22] 1. Ojima, "The Chemistry of Organic Silicon Compounds', Eds. S. Patai and Z. Rappoport, Wiley, New York, 1989, Part II, pp. 1479-1526, and ref. cit, therein.

[23] H. Bürgy, Doctoral Thesis, University of Bern, $199 \mathrm{~L}$.

[24] P. Bomhauser, G. Calzaferri, Spectrochim. Acta, Part A 1990, 46, 1045.

[25] M. Bärtsch, P. Bomhauser, G. Calzaferri, to be submitted.

[26] B. A. Kolesov, T. N. Martynova, T.I. Chupakhina, Zh. Strukt. 1988, 29, 77.

[27] F. J. Feher, D. A. Newman, J. F. Walzer, J. Am Chem. Soc. 1989, 1/1, 1741.

[28] E. J. J. Groenen, A. G. T. G. Kortbeeck, M. Mackay, O. Sudmeijer, Zeolites 1986, 6,403.

[29] E. J. J. Groenen, C. A. Emeis, J. P. Van den Berg, P. C. de Jong-Versloot, Zeolites 1987, 474.

[30] a) G. Calzaferri, M. Gori, Helv. Chim. Acta 1985 , 68,$1617 ;$ b) G. Calzaferri, M. Gori, H.R. Grüniger, W. Spahni, 'Proceedings Photoelectrochemistry: Fundamental Processes and Measurement 'Techniques, The Electrochemical Soc.', Eds. W. L. Wallace, A. J. Nozik, S. K. Deb, and R. H. Wilson, 1982, Vol.82-3, p. 264; c) H.R. Grüniger, G. Calzaferri, Helv. Chim. Acta 1979, 62, 2547.

[31] a) R. Beer, H. Bürgy, G. Calzaferri, I. Kamber, $J$ Electron. Spec. Rel. Phen. 1987, 44, 121; b) H. Bürgy, G. Calzaferri, 1. Kamber, Mikrochim. Acta 1988, 1, 401.

[32] N. J. Harrick, 'Internal Reflection Spectroscopy', John Wiley \& Sons, New York, 1967; F. M. Mirabella, Jr., N.J. Harrick, Harrick Scientific Corpor., Ossining, NY, 1985.
[33] J. Baumann, R. Beer, G. Calzaferri, B. Waldeck, J. Phys. Chem. 1989, 93, 2292.

[34] L. M. Hananaschvili, K. A. Adrianov, 'Technology of the Organoelement Monomers and Polymers, Chimia Moscow', 1983, pp. 293-295 (Russ.).

[35] W. M. Meier, D. H. Olson, Atlas of Zeolite Structure Types', 2nd edn., Butterworths, London, 1988.

[36] T. J. Meyer, Acc. Chem. Res. 1989, 22, 163.

[37] G. Calzaferri, L. Forss, W. Spahni, Chenic in unserer Zeit 1987, 2/, 161.

[38] a) G. Calzaferri, S. Hug, Th. Hugentobler, B. Sulzberger, J. Photochem. 1984, 26, 109; b) G. Calzaferri, W. Spahni, ibid. 1986, 32, 151; c) Chimia 1986, 40, 435; d) ibid. 1987, 41, 200

[39] G. Calzaferri, Proceedings of the 3 rd intemational summerschool, 'Solar Energy 88', Ed.G. Faninger, Kaprun/Austria, IFF, Klagenfurt, 1989, p. 119.

[40] A. Harriman, M. A. West, 'Photogeneration of Hydrogen', Academic Press, London, 1982.

[41] E. Amouyal, B. Zidler, Israel J. Chem. 1982, 22, 117.

[42] J. Hawecker, J.-M. Lehn, R.Ziessel,J.Chem.Soc., Chem. Commun. 1983, 536.

[43] G. Calzaferri, K. Hädener, J. Li, J. Chem. Soc., Chem. Commum., in press.

[44] G. Calzaferri, ESA SP-240 1985, 93.

[45] G. Calzaferri, J. Li, K. Hädener, N. Gfeller, D. Herren, M. Brändle, Eight International Conference on Photochemical Conversion and Storage of Solar Energy (I.P. S. 8), Palermo, Italy, 1990, Book of Abstracts, p. 212.
Chimia 45 (199/) 8-10

() Schweiz. Chemiker-Verband; ISSN 0009-4293

\section{Bestimmung von Selen in Lebensmitteln und in Mineralien mittels Neutronenaktivierungs- analysen}

\section{Marcel Langenauer und Urs Krähenbühl*}

Abstract. The determination of Se became important, since it was recognized that this trace element is essential for the human and animal diet. Lately, it was found that most patients suffering from a cancer disease manifest very low values of Se in their blood plasma. Activation analysis is a well suited technique to measure the concentrations of $S e$ in a vast range of materials with very different concentrations. At the lower end of concentrations, a radiochemical separation of Se following the irradiation is mandatory to obtain reliable results in the determination.

\section{Einleitung}

Selen galt seit Jangem als äusserst toxisches Element. Erst 1957 wurde seine essentielle Bedeutung für den Menschen erkannt
[1]. Durch Abgabe von Selentabletten an die Bevölkerung der Keshan-Region in China konnte die Kashin-Becksche Krankheit, eine rheumatisch-athritische Erkrankung, fast vollständig eliminiert werden. Heute ist be- kannt, dass bei vielen weiteren Krankheiten wie Krebs, Leukämie oder AIDS der SeGehalt im Blut einen Mangel aufzeigt. Es ist daher wichtig, dass die Bevölkerung genügend mit Se versorgt wird. Dieses Spurenelement kommt in Fleisch, Fisch und Getreideprodukten angereichert vor [2][3], wogegen es in Gemüse, Früchten und Milchprodukten verarmt ist [3]. Der tägliche Mindestbedarf des Menschen beträgt I $\mu \mathrm{g} \mathrm{Se} / \mathrm{kg}$ Körpergewicht [2]. Im allgemeinen sind die Böden der Schweiz recht arm an Se [4]. Importiertes Getreide aus den USA oder Kanada weist einen 10 bis 100 mal höheren Gehalt an diesem essentiellen Element auf als Weizen, der in der Schweiz geerntet wurde. Eine ausreichende Versorgung der Schweiz mit Se ist Dank der Getreideimporte gewährleistet. Die Entwicklung einer

*Korrespondenz: Prof. U. Krähenbühl Laboratorium für Radiochemie Universität Bern

Frejestrasse 3, CH-3000 Bern 9 
Tab. 1. Energieinterferenzen zu den ${ }^{75}$ Se $\tau$-Linien [5] (\%-Angabe: Linienhäufigkeit)

\begin{tabular}{lllc}
$\begin{array}{l}\tau-L \text { Linien von } \\
(T / 2=120.4[\mathrm{~d}]) \\
{[\mathrm{keV}]}\end{array}$ & $\begin{array}{l}\text { Interferenz } \\
\tau \text {-Linie } \\
{[\mathrm{keV}]}\end{array}$ & Radionuklid & Halbwertszeit \\
\hline $136.0(56 \%)$ & $136.2(6 \%)$ & ${ }^{181} \mathrm{Hf}$ & 42.5 \\
$264.6(59 \%)$ & $264.0(4 \%)$ & ${ }^{182} \mathrm{Ta}$ & 115.0 \\
$279.5(25 \%)$ & $279.2(82 \%)$ & $203 \mathrm{Hg}$ & 46.6 \\
\hline
\end{tabular}

Tab. 2. Ermittelte Se-Gehalte in Standardmaterialien (Fehler $\pm 1 \sigma$ )

\begin{tabular}{|c|c|c|c|c|}
\hline Standard & Materialart & $\begin{array}{l}\text { Bestimmte } \\
\text { RNAA-Gehalte } \\
\text { [ppb] }\end{array}$ & $\begin{array}{l}\text { Im Mittel } \\
\text { bestimmt } \\
\text { [ppb] }\end{array}$ & $\begin{array}{l}\text { Literatur } \\
\text { [ppb] }\end{array}$ \\
\hline NBS $1568^{a}$ ) & Reismehl & $\begin{array}{l}325 \pm 8 \\
320 \pm 8\end{array}$ & $323 \pm 6$ & $400 \pm 100$ \\
\hline BCR-189b) & Vollkorn & $\begin{array}{l}130 \pm 4 \\
128 \pm 3\end{array}$ & $129 \pm 3$ & $132 \pm 10$ \\
\hline$\left.M A-A-1^{C}\right)$ & $\begin{array}{l}\text { Schalentier- } \\
\text { Homogenat }\end{array}$ & $2950 \pm 70$ & & $3000 \pm 200$ \\
\hline$\left.H-4^{c}\right)$ & Tier Muskel & $285 \pm 7$ & & $280 \pm 30$ \\
\hline BHVO-1b) & Basalt & $120 \pm 10$ & . & $\begin{array}{l}95 \pm 34^{d} \\
74 \pm 44^{e}\end{array}$ \\
\hline
\end{tabular}

a) National Bureau of Standards (NBS) [6].

b) United States Geological Survey (USGS) [8].

c) International Atomic Energy Agency (IAEA) [5].

d) Nur AAS-Resultate aufgeführt [9].

e) Alle Resultate [9].
Tab. 3.: Se-Gehalte der untersuchten Prohen (Fehler $\pm 1 \sigma$ )

\begin{tabular}{|c|c|c|c|}
\hline \multicolumn{2}{|l|}{ Probe } & $\begin{array}{l}\text { Se-Gehalt } \\
\text { mittels RNAA } \\
\text { [ppb] }\end{array}$ & $\begin{array}{l}\text { Se-Gehalt } \\
\text { mittels INAA } \\
{[\mathrm{ppb}]}\end{array}$ \\
\hline \multicolumn{2}{|c|}{ Weizen Durum Kanada } & $1250 \pm 20$ & $1220 \pm 40$ \\
\hline \multicolumn{2}{|c|}{ Hartweizen Kleie } & $1230 \pm 20$ & $1150 \pm 40$ \\
\hline \multicolumn{4}{|c|}{ USA/Kanada } \\
\hline \multicolumn{2}{|c|}{ Hartweizenmehl } & $890 \pm 25$ & $880 \pm 45$ \\
\hline \multicolumn{4}{|c|}{ USA/Kanada } \\
\hline \multicolumn{2}{|c|}{ Weizen Frankreich } & $49 \pm 2$ & $10 \pm 9$ \\
\hline \multicolumn{2}{|c|}{ Weizen Hinwil (ZH) } & $30 \pm 2$ & n.n.") \\
\hline \multicolumn{2}{|c|}{ Weizen Chur (GR) } & $85 \pm 3$ & $80 \pm 10$ \\
\hline \multicolumn{2}{|c|}{ Weizen Giubiasco (Tl) } & $117 \pm 3$ & $119 \pm 5$ \\
\hline \multicolumn{2}{|c|}{ Weizen Gordola (TI) } & $62 \pm 2$ & $44 \pm 9$ \\
\hline \multicolumn{2}{|c|}{ Weizen Basel (BS) } & $47 \pm 4$ & $44 \pm 5$ \\
\hline \multicolumn{2}{|c|}{ Weizen Eiken (AG) } & $13 \pm 1$ & $\left.n, n,{ }^{a}\right)$ \\
\hline \multicolumn{2}{|c|}{ Roggen Chur (GR) } & $132 \pm 4$ & $110 \pm 10$ \\
\hline \multicolumn{2}{|c|}{ Roggen Basel (BS) } & $38 \pm 2$ & $7 \pm 5$ \\
\hline \multirow{2}{*}{\multicolumn{2}{|c|}{$\begin{array}{l}\text { Kakaomasse Westafrika } \\
\text { Kakaomasse Südamerika }\end{array}$}} & $185 \pm 6$ & $81 \pm 14$ \\
\hline & & $550 \pm 10$ & $580 \pm 50$ \\
\hline Probe & Mineral & $\begin{array}{l}\text { Se-Gehalt } \\
\text { mittels RNAA } \\
{[\mathrm{ppm}]}\end{array}$ & $\begin{array}{l}\text { Se-Gehalt } \\
\text { mittels INAA } \\
\text { [ppm] }\end{array}$ \\
\hline EP 88/1 & Baumhauerit & $50.2 \pm 1.0$ & $13.1 \pm 2.8$ \\
\hline EP $88 / 2$ & Baumhauerit & $50.5 \pm 1.0$ & $10.7 \pm 8$ \\
\hline EP $88 / 3$ & Baumhauerit & $890 \pm 20$ & $690 \pm 75$ \\
\hline EP 88/4 & Realgar & $86.6 \pm 1.8$ & $79.5 \pm 2.5$ \\
\hline EP $88 / 5$ & Pyrit & $2.18 \pm 0.05$ & $\left.n . n .{ }^{a}\right)$ \\
\hline EP $88 / 6$ & Pyrit & $2.34 \pm 0.05$ & n.n. $\left.{ }^{a}\right)$ \\
\hline
\end{tabular}

a) n.n.: nicht nachweisbar.
Nachweismethode für Se mit guter Empfindlichkeit und geringer Matrixabhängigkeit in verschiedensten Probenmaterialien war das Ziel dieser Arbeit. Die Neutronenaktivierungsanalyse erlaubt bei genügend hohen Gehalten, das gesuchte Element ohne chemisches Aufarbeiten zu messen. Für organisches Probenmaterial liegt diese Grenze bei einem Gehalt von $100 \mathrm{ppb}$. Die Hydrid-Atomabsorption-Spektroskopie liefert vergleichbare Resultate, solange der Gehalt nicht unter $50 \mathrm{ppb}$ absinkt. Oftmals können mit der Aktivierungsanalyse gleichzeitig weitere interessierende Elemente erfasst werden. Mit dem hier vorgestellten Verfahren kann mit geringer Anpassung auch Zinn bestimmt werden.

\section{Experimentelles}

Organische Proben wurden vorerst gefriergetrocknet. $50-100 \mathrm{mg}$ trockenes Probenmaterial wurden in Ampullen aus synthetischem Quarz eingeschmolzen und zusammen mit Referenzmaterial mit thermischen Neutronen während 1 Tag bei $3 \times 10^{13} \mathrm{n} \mathrm{cm}^{-2} \mathrm{~s}^{-1} \mathrm{im}$ Reaktor SAPHIR bestrahlt. Das durch die Bestrahlung erzeugte Nuklid ${ }^{75} \mathrm{Se}$ weist eine Halbwertszeit von 120 d auf und emittiert beim radioaktiven Zerfall Gammaquanten von 136, 264 und von $279 \mathrm{keV}$. In Tab. 1 sind die möglichen Interferenzenlinien und deren Häufigkeiten verzeichnet. Wenn das gesuchte Element zu keinem Zeitpunkt interferenzfrei gemessen werden konnte, wurde eine chemische Abtrennung vorgenommen. Zu diesem Zwecke musste das bestrahlte Probenmaterial in Gegenwart von $20 \mathrm{mg}$ Se-Träger aufgeschlossen werden. Angestrebt wurde ein Verfahren, welches verschiedene Aufschlussmethoden, aber identische Trenn- und Reinigungsschritte aufweist (Fig.). Um Verluste beim Aufschluss zu vermeiden, wurden diese in geschlossenen Teflon-Tiegeln vorgenommen. Organisches Probematerial wurde mit konz. $\mathrm{HNO}_{3}$ während $16 \mathrm{~h}$ bei einer Temp. von $100^{\circ}$ zersetzt. $\mathrm{Da}$ nach wurden die Proben mit $2 \mathrm{ml} \mathrm{HClO}_{4}$ versetzt und bis zum Aufsteigen von $\mathrm{HClO}_{4}$-Dämpfen erhitzt. Silikatproben wurden mit $2 \mathrm{ml} \mathrm{HF}, 1 \mathrm{ml} \mathrm{HClO}$ und $0.5 \mathrm{ml}$ $\mathrm{HNO}_{3}$ während $16 \mathrm{~h}$ bei $140^{\circ}$ aufgeschlossen. Nach dem Aufschluss wurden die Lsg. vollständig eingedampft. Die Rückstände wurden anschliessend mit 10 $\mathrm{ml} \mathrm{HCl}$ und $\mathrm{I} \mathrm{ml} \mathrm{HClO}_{4}$ in einen $100 \mathrm{ml}$ ZweihalsKolben übergeführt. Nun wurden $10 \mathrm{ml} \mathrm{HBr}$ zugegeben (alle verwendeten Säuren konzentriert) und das $\mathrm{SeBr}_{4}$ im $\mathrm{N}_{2}$-Strom (2-3 Blasen pro s) abdestilliert. Das Destillat wurde in $10 \mathrm{ml} 6 \mathrm{~m} \mathrm{HCl}$ aufgefangen. Durch Reduktion mittels $\mathrm{SO}_{2}$-Gas wird elementares Se ausgefailt und auf Glasfaserfilterscheiben abfiltriert. Die chemischen Ausbeuten betragen $96 \pm 4 \%$ für org. wie für anorg. Ausgangsmaterial. Die Messung der Gammalinien erfolgte mit einem Reingermaniumdetektor und die Auswertung wurde relativ zu der Aktivität eines Standards vorgenommen.

\section{Resultate und Diskussion}

In $T a b .2$ sind die radiochemisch ermittelten Se-Gehalte von Standardmaterialien zusammen mit Literaturdaten aufgeführt. Wir erkennen eine ausgezeichnete Übereinstimmung. Die Fehler der Resultate in dieser Arbeit sind systematisch kleiner als diejenigen anderer Autoren. Der in Tab. 2 und 3 angegebene Fehler umfasst $Z$ ählstatistik von Probe und Untergrund, den Gehaltfehler der hergestellten Standards und bei radiochemischen Proben die Unsicherheit der chemischen Ausbeute.

Tab. 3 enthält die Resultate der RNAA und der INAA der untersuchten Proben. Um die Se-Versorgung der Schweizer Bevölkerung durch Getreidekonsum abzuschätzen, wurden Proben aus verschiedenen Landesgegenden untersucht. Zudem standen uns auch Kakaomassen zur Verfügung. Die SeGehalte der untersuchten Weizenproben reflektieren die unterschiedliche Se-Verfügbarkeit in den verschiedenen Anbaugebieten. In der Schweiz finden wir in Proben, welche aus den Kantonen Tessin und Graubünden stammen, deutlich höhere Ge- 
halte. Weizen aus Frankreich zeigt einen ähnlichen Se Gehalt wie Proben aus dem Schweizerischen Mittelland. 10 bis $100 \mathrm{mal}$ grössere Gehalte finden sich in Proben aus Übersee. Die $25 \%$ aus Nordamerika importierten Getreide genügen, den täglichen Bedarf der Schweizer Bevölkerung an Se zu mehr als $50 \%$ zu decken. Die ergänzende Selenversorgung erfolgt vorwiegend über Fleisch- und Fischkonsum.

Die untersuchten Mineralien stammen alle aus dem Binntal (Wallis). Baumhauerit I und II stammen aus der gleichen Mineralisation. Es ist verständlich, dass Se als homologes Element von Schwefel in sulfidischen Erzen angereichert vorkommt. Der Unterschied des Se Gehaltes zwischen Baumhauerit $\left(\mathrm{Pb}_{12} \mathrm{As}_{16} \mathrm{~S}_{36}\right)$, Realgar $\left(\mathrm{As}_{4} \mathrm{~S}_{4}\right)$ und Pyrit $\left(\mathrm{FeS}_{2}\right)$ kann nicht erklärt werden. Es scheint, dass die As-haltigen Minerale generell reicher an Se sind. Gehalte von mehr als $100 \mathrm{ppb}$ Se können in Proben mit organischem Ursprung mit beiden Aktvierungsanalyseverfahren erfasst werden. Bei kleineren Gehalten aber liefert allein die radiochemische Bestimmung befriedigende Resultate. Mineralien zeigen nach einer Bestrahlung mit thermischen Neutronen eine wesentlich höhere Gesamtradioaktivität als organisches Material. Daher ist es verständlich, dass bei Mineralien die Grenze für das Erfassen des Se-Gehaltes mittels instrumenteller Aktivierungsanalyse höher liegt. Als Bestimmungsgrenze definieren wir nach Currie [10] den Wert, welcher noch mit kleinerem Fehler als $10 \%$ erfasst werden kann ( $\sigma$ Fehler, bei $50 \mathrm{mg}$ Probengewicht und $1 \mathrm{~d}$ Messdauer). Instrumentelle Aktivierungsanalyse von pflanzlichen Proben ergibt eine Bestimmungsgrenze für Selen von 100 $\mathrm{ppb}$, während für RNAA eine solche von 6 $\mathrm{ppb}$ angegeben werden kann. Die Nachweisgrenze (nach Currie [10]), die Grenze bei der qualitativ noch Se erkannt werden kann, liegt für organische Probenmaterialien bei $30 \mathrm{ppb}$ für INAA und bei $2 \mathrm{ppb}$ für RNAA. Auf Grund der gänzlich andern Zusammensetzung von Mineralproben liegen Nachweis- und Bestimmungsgrenzen für Se deutlich höher. Sie betragen für INAA 30 bzw. $100 \mathrm{ppm}$ für Baumhaueritminerale. Die Nachweis- und Bestimmungsgrenze von Se für radiochemisch aufgearbeitete Mineralproben liegt bei 12 bzw. $36 \mathrm{ppb}$.

\section{Schlussfolgerungen}

I) Der Se-Bedarf der Schweizer Bevölkerung wird vorallem durch das importierte Getreide aus Übersee gedeckt.

2) Die Aktivierungsanalyse erlaubt das Erfassen variabler Se-Gehalte in unterschiedlichsten Probenmaterialien.

3) Die Nachweisgrenzen liegen für instrumentelle Messungen bei $30 \mathrm{ppm}$ für Bleisulfide und bei $30 \mathrm{ppb}$ für planzliche Stoffe. Die entsprechenden Bestimmungsgrenzen betragen $100 \mathrm{ppm}$ für anorganische Proben und $100 \mathrm{ppb}$ für organische Proben.

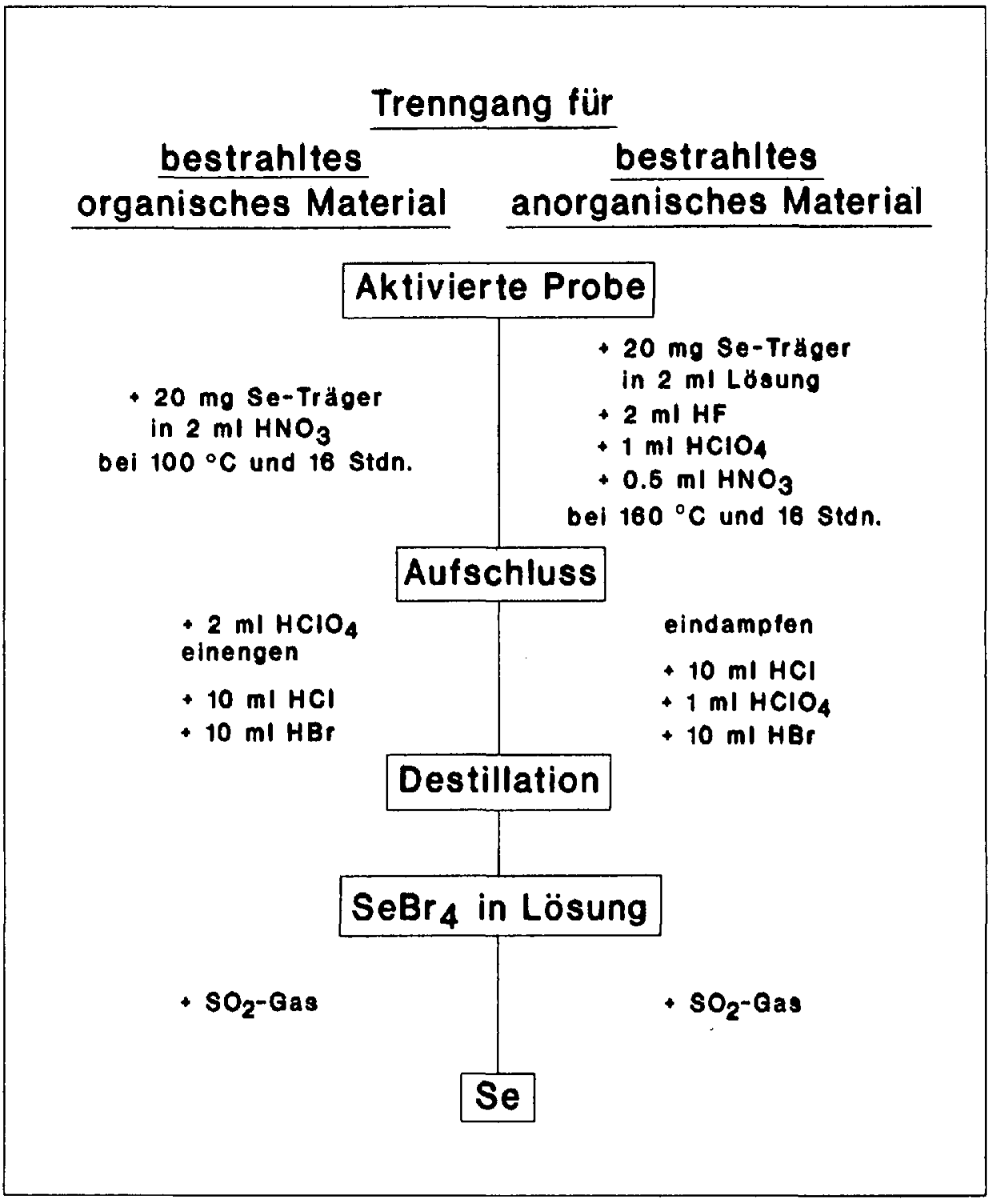

Figur. Schematischer Trenngang

4) Nach radiochemischem Abtrennen der Se-Aktiviät ergeben sich wesentlich tiefere Nachweisgrenzen von $12 \mathrm{ppb}$ für Minerale und $2 \mathrm{ppb}$ für Getreideproben. Die entsprechenden Bestimmungsgrenzen liegen bei 36 respektive $6 \mathrm{ppb}$ und gelten für Probengewichte von $50 \mathrm{mg}$.

Dem Bundesamtfür Gesundheitsw'esen danken wir für die bereitgestellten Getreideproben. Die Erzproben stammen aus dem Labor für Isotopengeologie. Wir danken $H . R$. von Gunten für sein Interesse an dieser Arbeit. Die Untersuchungen wurden durch Mittel des Schw'eizerischen Nationalfonds zur Fördertung der wissenschaftlichen Forschung unterstützt.
Eingegangen am 11. Dezember 1990

[1] K. Schwarz, C. M. Foltz, 'Selenium as an integral part of factor 3 against dietary necrotic liver degeneration', J. Am. Chem. Soc. 1957, 79, 3292.

[2] F. Kieffer, 'Selen, ein medizinisch bedeutungsvolles Spurenelement', Ars Medici 1987, 2, 60.

[3] J. J. Fardy, G. D. McOrist, Y. J. Farrar, 'The determination of selenium status in the australian diet using neutron activation analysis', I. Radionanal. Nucl. Chem., Articles 1989, Vol. 133. No. 2, 397405.

[4] Pilotstudie Abt. Isotopengeologie der Universität Bern, Vorkommen von Selen in Gesteinen, 1987.

[5] G. Erdimann, W. Soyka, 'The Gamma Rays of the Radionuclides', Verlag Chemie, Weinheim, 1979.

[6] National Bureau of Standards Certificate.

[7] IAEA Probenbeschreibung G4.12, 1989.

[8] E. S. Gladney and I. Roelandts, Geostandards Newslett. 1988, 12. 253.

[9] United States Geological Survey Certificate.

[10] L. A. Currie, 'Limits for Qualitative Detection and Quantitative Determination', Anal. Chem. $1968,40,586$. 